\title{
microRNAs involved in Parkinson's disease: A systematic review
}

\author{
FRANCIELE CASCAES DA SILVA ${ }^{1}$, RODRIGO DA ROSA IOP ${ }^{1}$, GIOVANNA GRUNEWALD VIETTA ${ }^{1}$, \\ DIEGO ALESSANDRO KAIR ${ }^{1}$, PAULO JOSÉ BARBOSA GUTIERRES FILHO ${ }^{2}$, \\ JOSÉ GUSTAVO SOUZA DE ALVARENGA ${ }^{2}$ and RUDNEY DA SILVA ${ }^{1}$ \\ ${ }^{1}$ Department of Physical Education, Center for Health Sciences and Sports, Adapted Physical Activity Laboratory, \\ University of State of Santa Catarina, Florianopolis 88080-350; ${ }^{2}$ Department of Physical Education, \\ Faculty of Physical Education, University of Brasilia, Brasilia 70910-900, Brazil
}

Received May 26, 2016; Accepted July 21, 2016

DOI: $10.3892 / \mathrm{mmr} .2016 .5759$

\begin{abstract}
The aim of the present study was to determine the expression of blood microRNAs (miRNAs) involved in PD in humans. For this purpose the following electronic databases were selected: MEDLINE by Pubmed, Scopus and Web of Science. The search strategy included the proposed descriptors in the Medical Subject Headings. There were no restrictions with respect to the language of the publication. In the study selection two independent reviewers initially evaluated studies that were identified by the search strategy according to titles and abstracts. The reviewers evaluated (also unassisted) the complete articles and selected studies according to the eligibility criteria specified above. Studies that were not in accordance with the adopted criteria were excluded according to the boundaries imposed by the search strategy. The following data were extracted from the selected studies: Publication identification, location where the study was conducted, study design, the sample size, the participants' characteristics, the miRNAs involved in PD, the miRNA detection and analysis method, and the type of miRNA dysregulation in PD. Through this systematic review of the literature published over the last 10 years, the expression of 91 different miRNAs were analyzed in the context of PD, with the expression of 39 of these miRNAs differing significantly between individuals with PD and healthy controls and/or between treated and untreated patients with PD. The miRNAs were extracted from mononuclear cells, leukocytes, plasma, serum and peripheral blood, and the majority of the studies used reverse transcription-quantitative polymerase chain reaction (RT-qPCR), which is considered to be the gold standard for miRNA analysis.
\end{abstract}

Correspondence to: Miss. Franciele Cascaes da Silva, Department of Physical Education, Center for Health Sciences and Sports, Adapted Physical Activity Laboratory, University of State of Santa Catarina, 358 Pascoal Simone Street, Coqueiros, Florianopolis 88080-350, Brazil

E-mail: francascaes@yahoo.com.br

Key words: Parkinson's disease, microRNAs, systematic review

\section{Introduction}

Parkinson's disease (PD) is the second most common neurodegenerative disease, and it is estimated to affect $\sim 1 \%$ of individuals $>60$ years of age and 4.1-4.6 million people worldwide (1). PD is a highly heterogeneous, progressive neurodegenerative disease, clinically characterized by tremors, bradykinesia, stiffness, and eventually postural instability (2). These symptoms may have variable progression (3) and are attributed to a loss of dopaminergic neurons in the substantia nigra (1). The pathology may involve other regions of the brain, including the amygdala, the cingulate gyrus and the superior cortical regions, resulting in the development of dementia and psychosis (1).

Previously, a number of reviews focusing on microRNAs (miRNAs) have demonstrated notable underlying molecular mechanisms corresponding to the pathogenesis of PD (1-7). It should be noted that the discovery of miRNAs broadened the potential of diagnostic markers and therapeutic targets for human diseases, influencing the majority of mechanisms responsible for neurodegenerative diseases (1), including PD (7).

miRNAs are small, endogenous, non-coding RNA molecules (length, 19-24 nt) that are cleaved from a partially duplexed 70-80-nt precursor, known as pre-miRNA (7). miRNAs regulate gene expression of the target mRNA at the post-transcriptional level via imperfect pairing with the 3'-untranslated region (3'-UTR). This affects numerous biological processes, including the cell cycle, differentiation, proliferation, apoptosis and the cellular stress response. Thus, as previous studies have suggested the importance of miRNAs in PD pathogenesis, and in the disturbance of processes associated with the regulation of genes implicated in its development (1-7), the aim of the present study was to determine the expression of blood miRNAs involved in PD in humans via a systematic review of the literature published over the last 10 years.

\section{Materials and methods}

Eligibility criteria. The present systematic review was registered on the International Prospective Register of Systematic Reviews (PROSPERO; no. CRD42015019973) and follows the 
recommendations proposed by the Preferred Reporting Items for Systematic Reviews and Meta-analyses: The PRISMA Statement (8).

Randomized and non-randomized studies of miRNAs in PD in humans where the miRNAs were obtained from blood samples were included. The previous studies were indexed on the previously selected databases, had abstracts available, were fully accessible online and were published within the past 10 years. There were no language restrictions.

Search strategy. The electronic databases MEDLINE via Pubmed (www.ncbi.nlm.nih.gov/pubmed), Scopus (Elsevier; www.scopus.com) and Web of Science (webofknowledge.com) were selected. The search strategy included the keywords proposed in medical subject headings (Table I). All the searches were conducted in September 2015. EndNote 3.4 (Thomson Reuters Corporation, Ontario, Canada) was used to manage reference material during searches.

Study selection and data extraction. The titles and abstracts of all of the articles identified by the search strategy were independently evaluated by two authors of the present study. These authors also evaluated the full articles and made their selections according to the pre-specified eligibility criteria. Disagreements between the reviewers were resolved by consensus.

The following data were extracted: Publication identification (first author and year of publication), location where the study was conducted (country), study design, the sample size, the participants' characteristics (gender, mean age, disease stage, mean disease duration), the miRNAs involved in PD, the miRNA detection and analysis method, and the type of miRNA dysregulation in PD.

\section{Results}

Literature search evaluated a total of 289 articles. The search enabled the identification of 289 articles, 76 of which were excluded as duplicates; 155 studies did not meet the eligibility criteria based on the titles and abstracts. A detailed evaluation indicated that nine studies were potentially relevant, and these were included in the review. Fig. 1 indicates the flowchart of this process.

Patient characteristics, analysis methods and miRNAs investigated were considered. The major characteristics of the studies included are described in Table II. Two studies were conducted in Spain $(9,10)$, and two were conducted in Italy $(11,12)$. The majority of the previous studies were cross-sectional (10-16). The sample size ranged from 7 (17) to 125 (10) individuals with PD. One study involved only male patients (17). The mean age of the participants ranged from 45 (13) to 72 (15) years. The PD stage (according to Hoehn and Yahr) ranged from $\mathrm{I}$ to $\mathrm{V}$, and the mean duration of the disease ranged from 3 (13) to 9.6 (10) years.

Table III indicates the miRNAs involved in PD, the detection and analysis methods, and the identified microRNA dysregulation in PD. The expression of 91 different miRNAs was observed. miRNAs were extracted from mononuclear cells (14), leukocytes (17), plasma $(9,15)$, serum $(10,11)$, and peripheral blood $(12,13,16)$. The majority of the analyses used reverse transcription-quantitative polymerase chain reaction (RT-qPCR), which is considered to be the gold standard analysis method.

miR-30b, miR-30c and miR-26a were downregulated in the previous study by Martins et al (14). Khoo et al (15) demonstrated that miR-1826, miR-626, and miR-505 were upregulated, and miR-450b-3p was downregulated. Upon comparison of patients with PD and healthy controls, Cardo et al (9) observed that miR-331-5p was elevated. Botta-Orfila et al (10), when comparing patients with idiopathic PD and controls, observed a downregulation of miR-29c, miR29-a and miR-19b. Vallelunga et al (11) observed a downregulation of miR-30c and miR-148b in patients with PD compared with patients with multiple system atrophy (MSA).

miR-1, miR-22* and miR-29a were downregulated and differed between patients with PD and healthy controls, and miR-16-2a*, miR-26a2* and miR-30a were upregulated with a significant difference between patients with treated and untreated PD (13). Serafin et al (12) demonstrated the patients treated with L-dopa exhibited decreased miR-103a-3p, miR-30b-5p and miR-29a-3p levels. Alieva et al (16) observed an upregulation of miR-7, miR-9-3p, miR-9-5p, miR-129 and miR-132 in patients with treated PD compared with untreated patients.

Comparing gene expression in patients with PD and healthy controls prior to and following electrical stimulation (deep brain stimulation-DBS) and $1 \mathrm{~h}$ after stimulus cessation, Soreq et al (17) observed that miR-1249, miR-20a, miR-18b, miR-378c and miR-4293 decreased following electrical stimulation, and $1 \mathrm{~h}$ after stimulus cessation, miR-423, miR-365, miR-486, miR-1260 and miR-218 were increased and miR-652, miR-15a*, miR-29c, miR-29a, miR-376c and were miR-143 decreased.

\section{Discussion}

The main aim of the present study was to determine the expression of miRNAs that are involved in human PD and present in the blood. The analysis consisted of a systematic review of the literature of the past 10 years, and in the nine studies considered, 91 different miRNAs were identified. Among these miRNAs, 39 were significantly differentially expressed between groups as follows; i) Individuals with PD and healthy controls $(9,10,14,15)$; ii) patients with PD and patients with systemic muscular atrophy (11); iii) patients with PD treated with levodopa and untreated PD $(12,13,16)$; and iv) patients who received electrical stimulation and those who did not (17).

Martins et al (14) demonstrated miR-30b, miR-30c and miR-26a were downregulated. According to these authors, these three miRNAs are involved in the regulation of $\alpha$-synuclein. The authors also discussed three common genes that are putative targets of these miRNAs, namely, ubiquitin specific peptidase (USP)6, E3 ubiquitin-protein ligase NEDD4 and USP3, and the roles of these proteins in glycosphingolipid biosynthesis and protein ubiquitination. Glycosphingolipids are related to ceramide metabolism, which has been associated with PD with regard to the glucocerebrosidase gene (18). Another important characteristic of PD is the presence of Lewy bodies and the ubiquitination of $\alpha$-synuclein, which is 
Table I. Descriptors used in the search strategy.

Topic Descriptors

MicroRNAs

Parkinson disease
'MicroRNAs' (MeSH), 'MicroRNAs', 'MicroRNA', 'miRNAs', 'Micro RNA', 'RNA, Micro', 'miRNA', 'Primary MicroRNA', 'MicroRNA, Primary', 'Primary miRNA', 'miRNA, Primary', 'pri-miRNA', 'pri miRNA', 'RNA, Small Temporal', 'Temporal RNA, Small', 'stRNA', 'Small Temporal RNA', 'pre-miRNA', 'pre miRNA

'Parkinson Disease' (MeSH), 'Parkinson Disease', 'Idiopathic Parkinson's Disease', 'Lewy Body Parkinson Disease', 'Lewy Body Parkinson's Disease', 'Primary Parkinsonism', 'Parkinsonism, Primary', 'Parkinson Disease, Idiopathic', 'Parkinson's Disease', 'Parkinson's Disease, Idiopathic', 'Parkinson's Disease, Lewy Body', 'Idiopathic Parkinson Disease', 'Paralysis Agitans'

MeSH, medical subject headings.

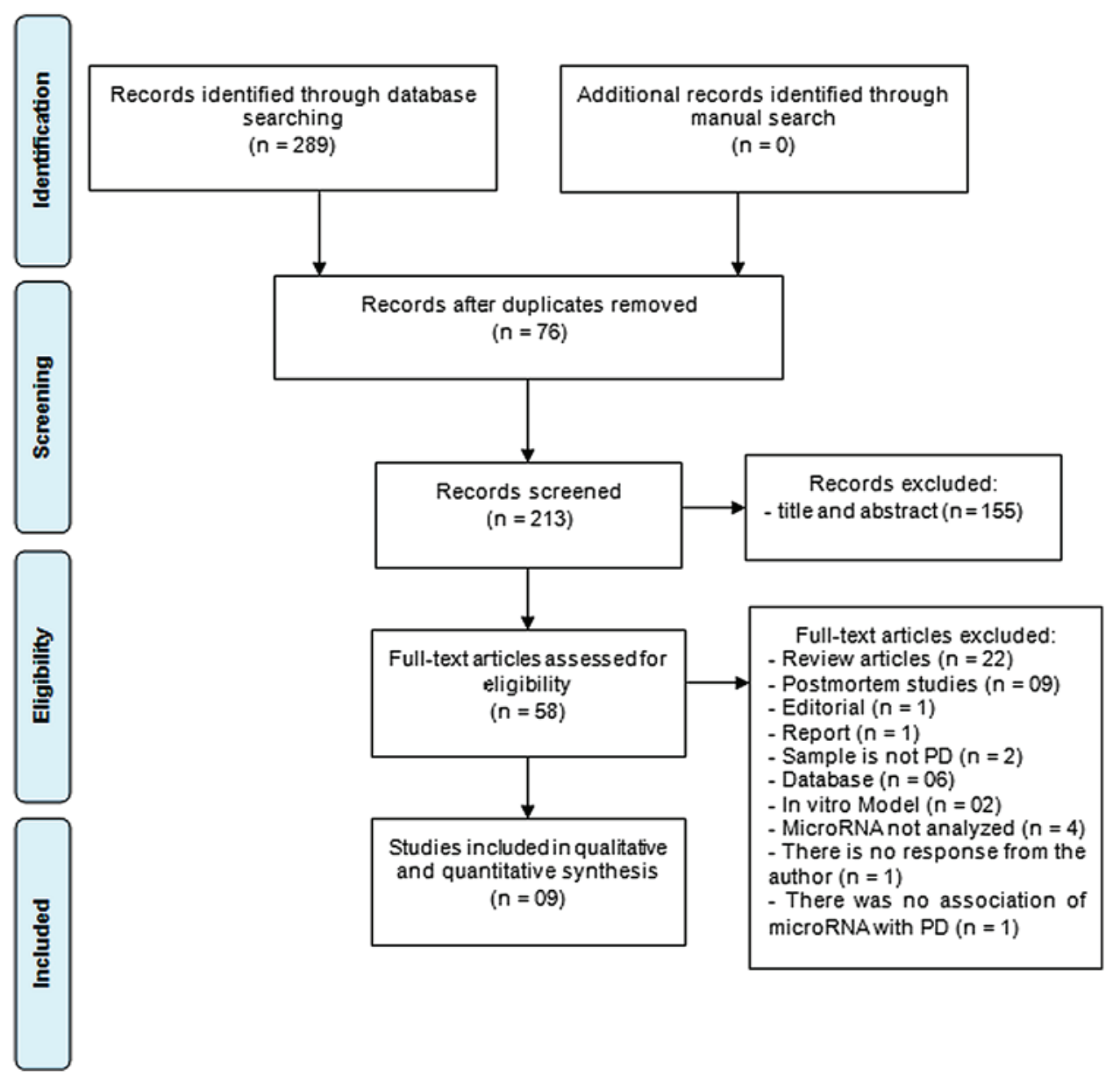

Figure 1. Flow diagram to summarize the search strategy. PD, Parkinson's disease; miRNA, microRNA.

a pathological event directly associated with the formation of Lewy bodies $(19,20)$.

Khoo et al (15) identified that miR-1826, miR-626, and miR-505 were upregulated and miR-450b-3p downregulated. miR-1826 was also observed to be upregulated in the plasma of patients with multiple sclerosis. This miRNA targets the neuronal PAS domain protein 3 gene and regulates neurogenesis, in particular in the hippocampus (21). miR-505 inhibits cell proliferation via induction of apoptosis (22) and regulates fibroblast growth factor (FGF)18, a pro-angiogenic factor (23).
However, dopamine depletion in PD is due to the apoptosis of dopaminergic neurons, an effect that may result from the increased apoptosis induction as a result of miR-505. Currently, no studies on miR-626 or miR-450b-3p have investigated their association with any disease or cell function, or have evaluated whether these biomarkers directly or indirectly function in PD pathogenesis (15).

When comparing patients with PD and healthy controls, Cardo et al (9) observed that miR-331-5p was significantly more elevated in the patients. The previous study also 


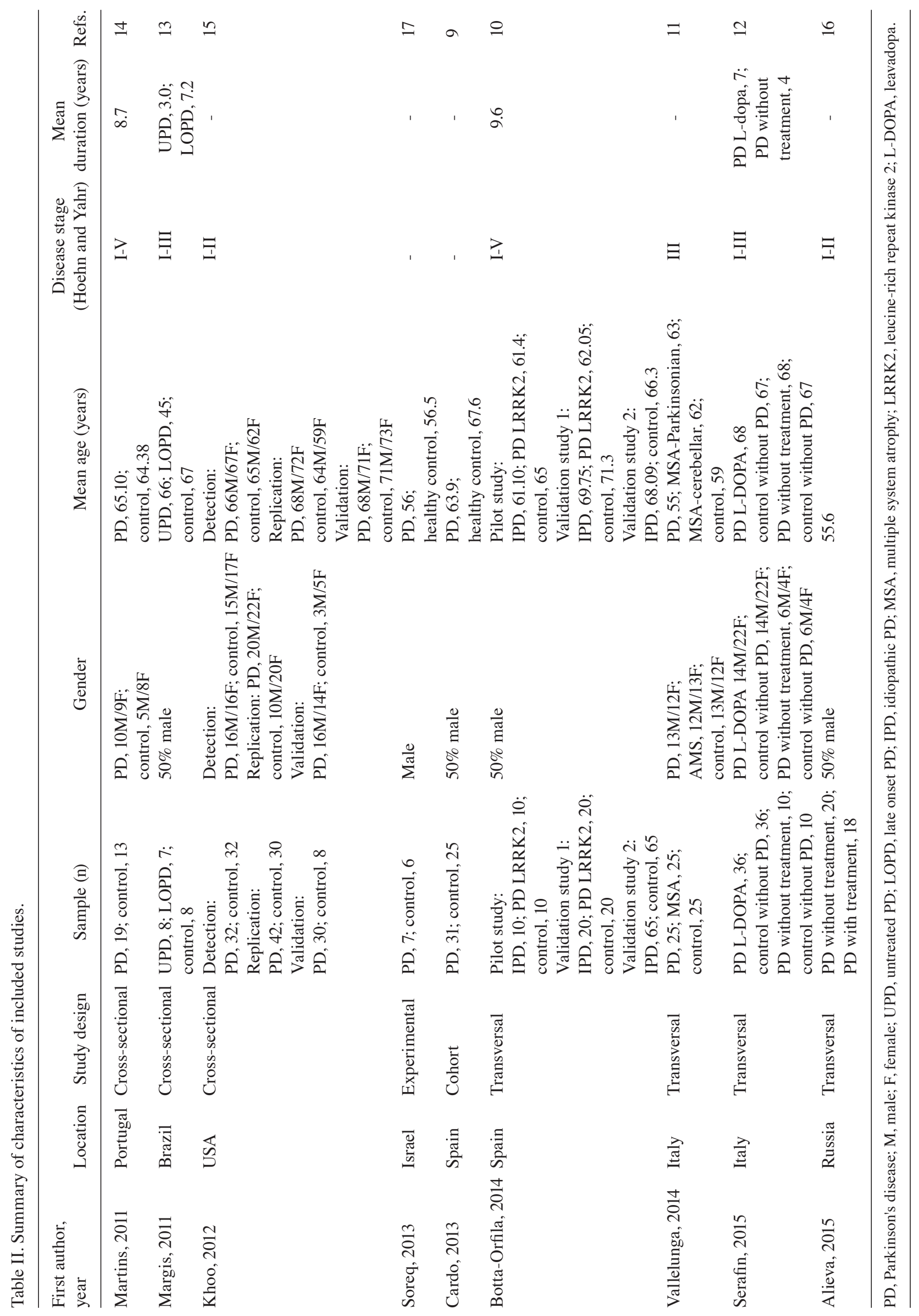


reported that this miRNA may be associated with neurogenesis and neurodegeneration and may be a useful biomarker of diagnosis, disease progression, and treatment response. Botta-Orfila et al (10) observed a downregulation of miR-29c, miR-29a and miR-19b when patients were compared with idiopathic PD and controls. Fernández-Santiago et al (24) indicated that miR-19b dysregulation occurs in the prodromal phase of synucleinopathies. The accumulation of $\alpha$-synuclein in the form of intracellular filamentous aggregates is one of the pathological features of the neurodegenerative diseases designated synucleinopathies, which include PD (25). Vallelunga et al (11) observed a downregulation of miR-30c and miR-148b in patients with PD compared with patients with MSA. miR-148b is important in neurological development and apoptosis (26).

The levels of miR-1, miR-22* and miR-29a were lower in untreated patients than in healthy individuals, whereas the levels of miR-16-2a*, miR-26a2* and miR-30a were higher in patients treated with levodopa than in untreated patients (13). These miRNAs may be indirectly associated with the functioning of the $\alpha$-synuclein gene, synuclein- $\alpha$ (SNCA), which is responsible for coding the $\alpha$-synuclein protein (5). SNCA is one of the primary genes implicated in the development of PD (27). The specific role of this protein remains to be elucidated, however, data has suggested that it is a molecular chaperone that regulates protein-protein and protein-lipid interactions, and that it may be important in the metabolism of synaptic vesicles and the storage and compartmentalization of neurotransmitters, particularly dopamine (28). High SNCA expression is characteristic of PD (29).

miR-1 and miR-30a are involved in the regulation of dopamine transport and are therefore implicated in the pathogenesis of PD (5). Furthermore, miR-30 may bind FGF20, which is also associated with miR-16-2a* (13). FGF20 is preferentially expressed in the substantia nigra and stimulates dopaminergic neuron maturation (30). These results would explain the correlation observed between high SNCA expression and high FGF20 translation in cultures of brain cells and tissues from patients with PD (30).

Margis and Rieder (13) demonstrated miR-29a was the only miRNA which exhibited reduced expression following treatment with levodopa. The authors suggest that miR-29a expression may not be directly associated with the occurrence of motor symptoms given that downregulation was maintained following administration of the therapeutic agent. However, the authors highlighted the importance of miR-29a in PD.

Cell division cycle 42 is a protein associated with septin 4 , and a target of miR-29a. Shehadeh et al (31) observed that in addition to increased septin 4 levels, $\alpha$-synuclein levels were also increased in the substantia nigra post mortem in patients with PD. Decreased miR-29a expression also occurs in Alzheimer's disease in association with an increase in $\beta$-secretase (BACE1) $(32,33)$. The levels of insulin-like growth factor 1 (IGF-1) are associated with the preservation and homeostasis of the nervous system, and IGF-1 is also a target of miR-29a. IGF-1 levels in the serum and brain differ in a number of human neurodegenerative diseases (34). A previous study demonstrated that blood IGF-1 levels were slightly elevated in treated patients with PD compared with controls, although this effect was not statistically significant (35).
Eacker et al (36) demonstrated a significant association between lower miR-29a expression and higher BACE1 expression in brain tissue. miR-29a is expressed in neurons and astroglia, suggesting that the specific reductions in its expression are not a secondary consequence of the death of specific neurons.

Serafin et al (12) observed that patients treated with levodopa exhibited decreased levels of miR-103a-3p, miR-30b-5p and miR-29a-3p. The results suggest a role of the treatment with levodopa for the expression of miR-103a-3p, indicating a potential association with the occurrence of motor symptoms. Other studies have demonstrated an association between miRNAs 103a-3p and 29a-3p and insulin resistance, a process that may be associated with neurodegeneration in PD $(37,38)$.

Alieva et al (16) observed an upregulation of miR-7, miR-9-3p, miR-9-5p, miR-129 and miR-132 in treated patients with PD when compared with untreated patients. The authors hypothesize that miRNAs are sensitive to therapy and that an effect of treatment may be observed in changes in their levels as well as those of their target genes in patients with PD. In addition to administration of therapeutic agents, other factors may influence miRNA expression, such as the presence of comorbidities, disease severity, disease duration, and gender $(10,15)$.

miR-7 is abundantly expressed in the brain and binds preferentially to the 3'UTR of the SNCA gene, which significantly decreases the synthesis of $\alpha$-synuclein and inhibits $\alpha$-synuclein-mediated cell death (6). miR-9-3p was identified as a tumor suppressor in hepatocellular carcinoma (39). miR-9-5p has an inhibitory effect on transforming growth factor beta receptor type II and on the expression of NADPH-oxidase 4 (40). The ectopic expression of miR-129 promoted apoptosis, cell proliferation and cell cycle inhibition in colorectal cancer cells (41). miR-132 is enriched in the central nervous system and may be involved in neuron development, maturation, and function (42).

By comparing gene expression in patients with PD and healthy controls prior to and following DBS and $1 \mathrm{~h}$ after stimulus cessation, Soreq et al (17) observed that miR-1249, miR-20a, miR-18b, miR-378c and miR-4293 were reduced following electrical stimulation, and $1 \mathrm{~h}$ after stimulus cessation, miR-423, miR-365, miR-486, miR-1260 and miR-218 were increased and miR-652, miR-15a*, miR-29c, miR-29a, miR-376c and miR-143 were decreased.

miR-365 is a negative regulator of interleukin-6 (IL-6) (43). A cohort study involving 53 patients with PD demonstrated that high levels of IL-6 may be a marker of mortality in patients with PD (44). Scalzo et al (45) demonstrated that high levels of IL-6 may be involved in the acceleration of muscle catabolism, leading to sarcopenia, and thus may contribute to weakness and fatigue and be associated with functional impairment in PD.

Previous studies have associated miR-20a, miR-18b, miR-365, miR-486, miR-218, miR-652, miR-15a*, miR-29c, miR-376c, and miR-143 with hepatocellular carcinoma $(46,47)$, breast cancer (48), liver disease (49), lung cancer (50), and melanoma (51), but these miRNAs were not associated with PD.

The miRNAs in the reviewed previous studies were extracted from mononuclear cells, leukocytes, plasma, serum and peripheral blood. Thus, it has been demonstrated that blood 


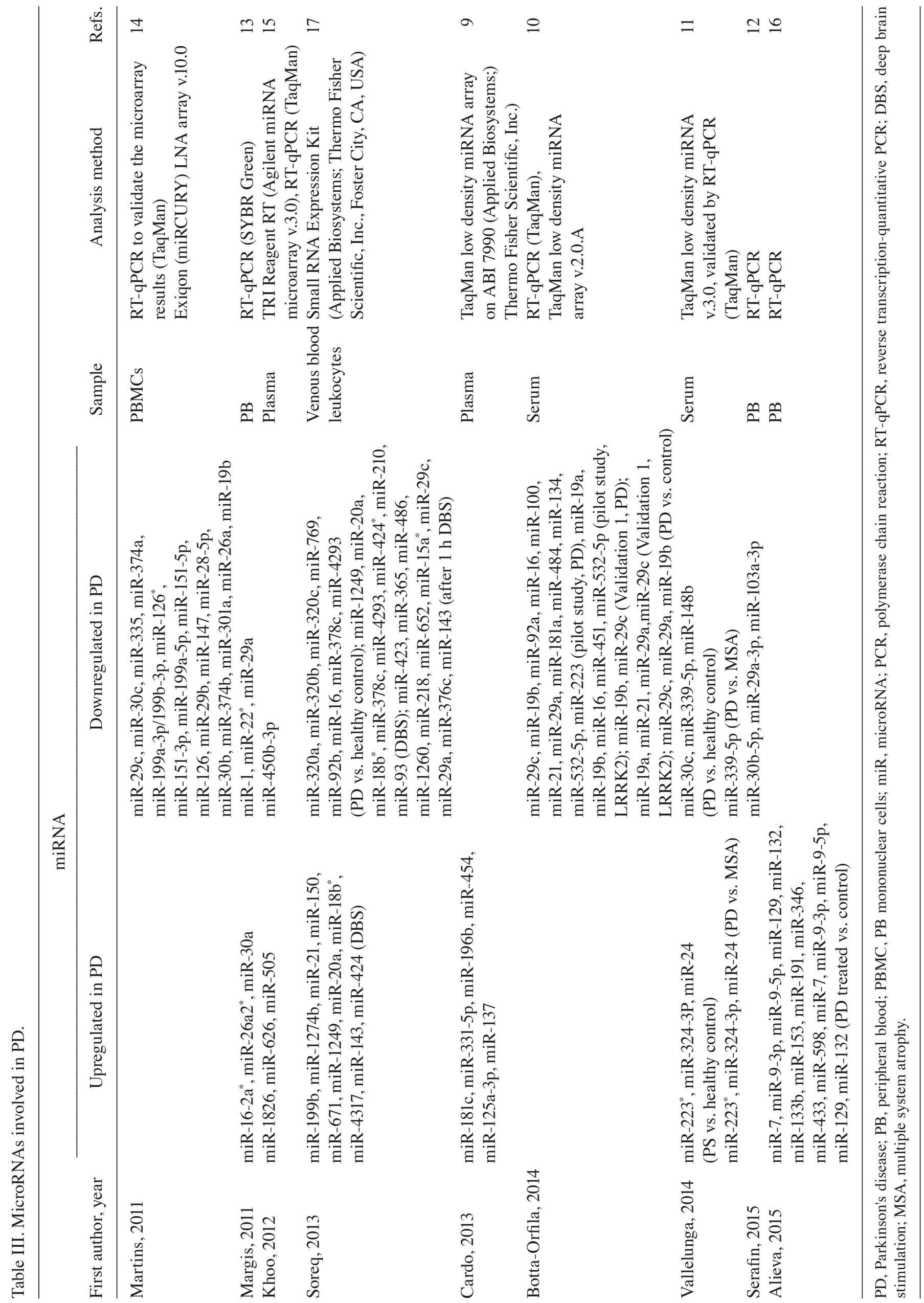


samples are a useful source in the identification of miRNAs associated with PD. Lawrie et al (52) were the first to investigate the presence of miRNAs in serum, comparing patients with diffuse lymphoma and healthy individuals. Another study has demonstrated the presence of miRNAs in other biological fluids, including urine, saliva, amniotic fluid, and pleural fluid (53).

An ideal biomarker must be specific, sensitive, minimally invasive, quantifiable, cost-effective and reproducible. Therefore, blood analysis may provide an ideal resource for the development of biomarkers that fit these requirements and enable patient monitoring over time. By contrast, a biopsy for PD is almost impossible, and neuroimaging every three months is financially prohibitive (15). The use of miRNAs as biomarkers has certain advantages. miRNAs are easy to detect, and the methods are extremely specific. Furthermore, as reliable data analysis is essential to the outcome of a given study, it is important to select adequate normalization methods to remove variations and to increase precision in miRNA quantification (54).

The miRNA analysis used in the majority of the reviewed studies was RT-qPCR. The RT-qPCR technique is the gold standard in nucleic acid quantification, due to its sensitivity and specificity (55). Briefly, the procedure involves four major steps: i) Sample collection and preparation; ii) the generation of global miRNA profiles using RT-qPCR; iii) data normalization and analysis; and iv) miRNA biomarker selection and validation (56).

In summary, there is a general lack of studies that confirm the involvement in PD of the majority of miRNAs presented in the reviewed studies. Novel analyses are important to establish the expression profile of miRNAs in PD in order to more accurately determine the ideal treatment strategy and to improve the role of miRNAs as biomarkers in clinical practice.

In conclusion, this systematic review of literature from the past 10 years analyzed the expression of 91 different miRNAs in the context of PD, with the expression of 39 of these miRNAs differing significantly between individuals with PD and healthy controls and/or between treated and untreated patients with PD. The downregulated miRNAs were as follows: miR-30b, miR-30c, miR-26a, miR-450b-3p, miR-148b, miR-1, miR-22*, miR-29a, miR-103a-3p, miR-30b-5p, miR-29a-3p, miR-1249, miR-20a, miR-18b, miR-378c, miR-4293, miR-652, miR-15a*, miR-29c, miR-376c, miR-143, and miR-19b. The upregulated miRNAs were as follows: miR-1826, miR-626, miR-505, miR-16-2a*, miR-26a2*, miR-30a, miR-7, miR-9-3-p, miR-9-5p, miR-129, miR-132, miR-423, miR-365, miR-486, miR-1260, miR-218, and miR-331-5p. The miRNAs were extracted from mononuclear cells, leukocytes, plasma, serum and peripheral blood, and the majority of the studies used RT-qPCR, considered to be the gold standard, for miRNA analysis.

\section{Acknowledgements}

The authors would like thank the Coordination of Improvement of Higher Education Personnel of the Ministry of Education of Brazil and the University of Brasilia (Brasilia, Brazil).

\section{References}

1. Maciotta S, Meregalli M and Torrente Y: The involvement of microRNAs in neurodegenerative diseases. Front Cell Neurosci 7: 265, 2013.
2. Shtilbans A and Henchcliffe C: Biomarkers in Parkinson's disease: An update. Curr Opin Neurol 25: 460-465, 2012.

3. Mouradian MM: MicroRNAs in Parkinson's disease. Neurobiol Dis 46: 279-284, 2012.

4. Harraz MM, Dawson TM and Dawson VL: MicroRNAs in Parkinson's disease. J Chem Neuroanat 42: 127-130, 2011.

5. Filatova EV, Alieva AK, Shadrina MI and Slominsky PA: MicroRNAs: Possible role in pathogenesis of Parkinson's disease. Biochemistry (Mosc) 77: 813-819, 2012.

6. Junn E and Mouradian MM: MicroRNAs in neurodegenerative diseases and their therapeutic potential. Pharmacol Ther 133: 142-150, 2012.

7. Wang C, Ji B, Cheng B, Chen J and Bai B: Neuroprotection of microRNA in neurological disorders (Review). Biomed Rep 2: 611-619, 2014.

8. Moher D, Liberati A, Tetzlaff $\mathrm{J}$ and Altman DG; PRISMA Group: Preferred reporting items for systematic reviews and meta-analyses: The PRISMA statement. Int J Surg 8: 336-341, 2010.

9. Cardo LF, Coto E, de Mena L, Ribacoba R, Moris G, Menéndez M and Alvarez V: Profile of microRNAs in the plasma of Parkinson's disease patients and healthy controls. J Neurol 260: 1420-1422, 2013.

10. Botta-Orfila T, Morató X, Compta Y, Lozano JJ, Falgàs N, Valldeoriola F, Pont-Sunyer C, Vilas D, Mengual L, Fernández M, et al: Identification of blood serum micro-RNAs associated with idiopathic and LRRK2 Parkinson's disease. J Neurosci Res 92: 1071-1077, 2014.

11. Vallelunga A, Ragusa M, Di Mauro S, Iannitti T, Pilleri M, Biundo R, Weis L, Di Pietro C, De Iuliis A, Nicoletti A, et al: Identification of circulating microRNAs for the differential diagnosis of Parkinson's disease and multiple system atrophy. Front Cell Neurosci 8: 156, 2014.

12. Serafin A, Foco L, Zanigni S, Blankenburg H, Picard A, Zanon A, Giannini G, Pichler I, Facheris MF, Cortelli P, et al: Overexpression of blood microRNAs 103a, 30b, and 29a in L-dopa-treated patients with PD. Neurology 84: 645-653, 2015.

13. Margis R and Rieder CR: Identification of blood microRNAs associated to Parkinsonǐs disease. J Biotechnol 152: 96-101, 2011.

14. Martins M, Rosa A, Guedes LC, Fonseca BV, Gotovac K, Violante S, Mestre T, Coelho M, Rosa MM, Martin ER, et al: Convergence of miRNA expression profiling, $\alpha$-synuclein interacton and GWAS in Parkinson's disease. PLoS One 6: e25443, 2011.

15. Khoo SK, Petillo D, Kang UJ, Resau JH, Berryhill B, Linder J, Forsgren L, Neuman LA and Tan AC: Plasma-based circulating microRNA biomarkers for Parkinson's disease. J Parkinsons Dis 2: 321-331, 2012.

16. Alieva AK, Filatova EV, Karabanov AV, Illarioshkin SN, Limborska SA, Shadrina MI and Slominsky PA: miRNA expression is highly sensitive to a drug therapy in Parkinson's disease. Parkinsonism Relat Disord 21: 72-74, 2015

17. Soreq L, Salomonis N, Bronstein M, Greenberg DS, Israel Z, Bergman $\mathrm{H}$ and Soreq $\mathrm{H}$ : Small RNA sequencing-microarray analyses in Parkinson leukocytes reveal deep brain stimulation-induced and splicing changes that classify brain region transcriptomes. Front Mol Neurosci 6: 10, 2013.

18. Sidransky E, Nalls MA, Aasly JO, Aharon-Peretz J, Annesi G, Barbosa ER, Bar-Shira A, Berg D, Bras J, Brice A, et al: Multicenter analysis of glucocerebrosidase mutations in Parkinson's disease. N Engl J Med 361: 1651-1661, 2009.

19. Hasegawa M, Fujiwara H, Nonaka T, Wakabayashi K, Takahashi H, Lee VM, Trojanowski JQ, Mann D and Iwatsubo T: Phosphorylated alpha-synuclein is ubiquitinated in alpha-synucleinopathy lesions. J Biol Chem 277: 49071-49076, 2002.

20. Tofaris GK, Razzaq A, Ghetti B, Lilley KS and Spillantini MG: Ubiquitination of alpha-synuclein in Lewy bodies is a pathological event not associated with impairment of proteasome function. J Biol Chem 278: 44405-44411, 2003.

21. Siegel SR, Mackenzie J, Chaplin G, Jablonski NG and Griffiths L: Circulating microRNAs involved in multiple sclerosis. Mol Biol Rep 39: 6219-6225, 2012.

22. Yamamoto Y, Yoshioka Y, Minoura K, Takahashi RU, Takeshita F, Taya T, Horii R, Fukuoka Y, Kato T, Kosaka N and Ochiya T: An integrative genomic analysis revealed the relevance of microRNA and gene expression for drug-resistance in human breast cancer cells. Mol Cancer 10: 135, 2011.

23. Yang Q, Jia C, Wang P, Xiong M, Cui J, Li L, Wang W, Wu Q, Chen Y and Zhang T: MicroRNA-505 identified from patients with essential hypertension impairs endothelial cell migration and tube formation. Int J Cardiol 177: 925-934, 2014. 
24. Fernández-Santiago R, Iranzo A, Gaig C, Serradell M, Fernández M, Tolosa E, Santamaría J and Ezquerra M: MicroRNA association with synucleinopathy conversion in rapid eye movement behavior disorder. Ann Neurol 77: 895-901, 2015.

25. Goedert M: Parkinson's disease and other alpha-synucleinopathies. Clin Chem Lab Med 39: 308-312, 2001.

26. Murad N, Kokkinaki M, Gunawardena N, Gunawan MS, Hathout Y, Janczura KJ, Theos AC and Golestaneh N: miR-184 regulates ezrin, LAMP-1 expression, affects phagocytosis in human retinal pigment epithelium and is downregulated in age-related macular degeneration. FEBS J 281: 5251-5264, 2014.

27. Fortin DL, Troyer MD, Nakamura K, Kubo S, Anthony MD and Edwards RH: Lipid rafts mediate the synaptic localization of alpha-synuclein. J Neurosci 24: 6715-6723, 2004.

28. Yavich L, Tanila H, Vepsäläinen S and Jäkälä P: Role of alpha-synuclein in presynaptic dopamine recruitment. J Neurosci 24: 11165-11170, 2004.

29. Doxakis E: Post-transcriptional regulation of alpha-synuclein expression by mir-7 and mir-153. J Biol Chem 285: 12726-12734, 2010.

30. Wang G, van der Walt JM, Mayhew G,Li YJ,Züchner S, Scott WK, Martin ER and Vance JM: Variation in the miRNA-433 binding site of FGF20 confers risk for Parkinson disease by overexpression of alpha-synuclein. Am J Hum Genet 82: 283-289, 2008

31. Shehadeh L, Mitsi G, Adi N, Bishopric N and Papapetropoulos S: Expression of Lewy body protein septin 4 in postmortem brain of Parkinson's disease and control subjects. Mov Disord 24 204-210, 2009.

32. Hébert SS, Horré K, NicolaïL,Papadopoulou AS, Mandemakers W, Silahtaroglu AN, Kauppinen S, Delacourte A and De Strooper B: Loss of microRNA cluster miR-29a/b-1 in sporadic Alzheimer's disease correlates with increased BACE1/beta-secretase expression. Proc Natl Acad Sci USA 105: 6415-6420, 2008.

33. Shioya M, Obayashi S, Tabunoki H, Arima K, Saito Y, Ishida T and Satoh J: Aberrant microRNA expression in the brains of neurodegenerative diseases: miR-29a decreased in Alzheimer disease brains targets neurone navigator 3. Neuropathol Appl Neurobiol 36: 320-330, 2010.

34. Busiguina S, Fernandez AM, Barrios V, Clark R, Tolbert DL, Berciano $\mathrm{J}$ and Torres-Aleman I: Neurodegeneration is associated to changes in serum insulin-like growth factors. Neurobiol Dis 7: 657-665, 2000.

35. Tuncel D, Inanc Tolun F and Toru I: Serum insulin-like growth factor-1 and nitric oxide levels in Parkinson's disease. Mediators Inflamm 2009: 132464, 2009.

36. Eacker SM, Dawson TM and Dawson VL: Understanding microRNAs in neurodegeneration. Nat Rev Neurosci 10 837-841, 2009

37. Trajkovski M, Hausser J, Soutschek J, Bhat B, Akin A, Zavolan M, Heim MH and Stoffel M: MicroRNAs 103 and 107 regulate insulin sensitivity. Nature 474: 649-653, 2011.

38. Aviles-Olmos I, Limousin P, Lees A and Foltynie T: Parkinson's disease, insulin resistance and novel agents of neuroprotection. Brain 136: 374-384, 2013

39. Higashi T, Hayashi H, Ishimoto T, Takeyama H, Kaida T, Arima K, Taki K, Sakamoto K, Kuroki H, Okabe H, et al: miR-9-3p plays a tumour-suppressor role by targeting TAZ (WWTR1) in hepatocellular carcinoma cells. Br J Cancer 113: 252-258, 2015.

40. Fierro-Fernández M, Busnadiego Ó, Sandoval $\mathrm{P}$, Espinosa-Díez C, Blanco-Ruiz E, Rodríguez M, Pian H, Ramos R, López-Cabrera M, García-Bermejo ML and Lamas S: miR-9-5p suppresses pro-fibrogenic transformation of fibroblasts and prevents organ fibrosis by targeting NOX4 and TGFBR2. EMBO Rep 16: 1358-1377, 2015.
41. Karaayvaz M, Zhai H and Ju J: miR-129 promotes apoptosis and enhances chemosensitivity to 5 -fluorouracil in colorectal cancer. Cell Death Dis 4: e659, 2013.

42. Zhang S, Hao J, Xie F, Hu X, Liu C, Tong J, Zhou J, Wu J and Shao C: Downregulation of miR-132 by promoter methylation contributes to pancreatic cancer development. Carcinogenesis 32 1183-1189, 2011.

43. Xu Z, Xiao SB, Xu P, Xie Q, Cao L, Wang D, Luo R, Zhong Y, Chen HC and Fang LR: miR-365, a novel negative regulator of interleukin- 6 gene expression, is cooperatively regulated by $\mathrm{Sp} 1$ and NF-kappaB. J Biol Chem 286: 21401-21412, 2011.

44. Dufek M, Rektorova I, Thon V, Lokaj J and Rektor I: Interleukin-6 May contribute to mortality in Parkinson's disease patients: A 4-year prospective study. Parkinsons Dis 2015: 898192, 2015.

45. Scalzo P, Kümmer A, Cardoso F and Teixeira AL: Serum levels of interleukin-6 are elevated in patients with Parkinson's disease and correlate with physical performance. Neurosci Lett 468: $56-58,2010$.

46. Fan MQ, Huang CB, Gu Y, Xiao Y, Sheng JX and Zhong L: Decrease expression of microRNA-20a promotes cancer cell proliferation and predicts poor survival of hepatocellular carcinoma. J Exp Clin Cancer Res 32: 21, 2013.

47. Tu K, Li C, Zheng X, Yang W, Yao Y and Liu Q: Prognostic significance of miR-218 in human hepatocellular carcinoma and its role in cell growth. Oncol Rep 32: 1571-1577, 2014.

48. Leivonen SK, Mäkelä R, Ostling P, Kohonen P, Haapa-Paananen S, Kleivi K, Enerly E, Aakula A, Hellström K, Sahlberg N, et al: Protein lysate microarray analysis to identify microRNAs regulating estrogen receptor signaling in breast cancer cell lines. Oncogene 28: 3926-3936, 2009.

49. Roderburg C, Mollnow T, Bongaerts B, Elfimova N, Vargas Cardenas D, Berger K, Zimmermann H, Koch A, Vucur M, Luedde M, et al: Micro-RNA profiling in human serum reveals compartment-specific roles of miR-571 and miR-652 in liver cirrhosis. PLoS One 7: e32999, 2012.

50. Wei J, Ma Z, Li Y, Zhao B, Wang D, Jin Y and Jin Y: miR-143 inhibits cell proliferation by targeting autophagy-related $2 \mathrm{~B}$ in non-small cell lung cancer H1299 cells. Mol Med Rep 11: 571-576, 2015.

51. Zehavi L, Avraham R, Barzilai A, Bar-Ilan D, Navon R, Sidi Y, Avni D and Leibowitz-Amit R: Silencing of a large microRNA cluster on human chromosome 14q32 in melanoma: Biological effects of mir-376a and mir-376c on insulin growth factor 1 receptor. Mol Cancer 11: 44, 2012.

52. Lawrie CH, Gal S, Dunlop HM, Pushkaran B, Liggins AP, Pulford K, Banham AH, Pezzella F, Boultwood J, Wainscoat JS, et al: Detection of elevated levels of tumour-associated microRNAs in serum of patients with diffuse large B-cell lymphoma. Br J Haematol 141: 672-675, 2008.

53. Gilad S, Meiri E, Yogev Y, Benjamin S, Lebanony D, Yerushalmi N, Benjamin $\mathrm{H}$, Kushnir M, Cholakh $\mathrm{H}$, Melamed N, et al: Serum microRNAs are promising novel biomarkers. PLoS One 3: e3148, 2008.

54. Grasso M, Piscopo P, Confaloni A and Denti MA: Circulating miRNAs as biomarkers for neurodegenerative disorders. Molecules 19: 6891-6910, 2014.

55. Mestdagh P, Van Vlierberghe P, De Weer A, Muth D, Westermann F, Speleman F and Vandesompele J: A novel and universal method for microRNA RT-qPCR data normalization. Genome Biol 10: R64, 2009.

56. Kang K, Peng X, Luo J and Gou D: Identification of circulating miRNA biomarkers based on global quantitative real-time PCR profiling. J Anim Sci Biotechnol 3: 4, 2012. 